media and the DTC test have shown higher sensitivities, $98 \%{ }^{5}$ and $86.2 \%,{ }^{10}$ figures comparable with our own results. Furthermore, a two-hour reading is as reliable as a test read at six or 24 hours' incubation. ${ }^{510}$ Staphylococci cultured from screen negative blood culture broths should, however, be re-tested for free coagulase and/or clumping factor/protein A plus DNAse.

If the DTC test is routinely combined with tests for clumping factor/protein A and DNAse from plate cultures, mis-identification of coagulase-positive staphylococci should be avoided. Staphylococcus intermedius, a potentially invasive pathogen from dog-bite wounds, is usually tube coagulase positive (rabbit plasma positive, human plasma variable), clumping factor/protein A negative but DNAse positive. Staphylococcus cugdunensis, associated with a variety of infections including endocarditis, is typically tube coagulase negative, clumping factor positive and weakly DNAse positive. $S$ aureus should be positive for all three tests.

Of other potential rapid diagnostic methods for $S$ aureus bacteraemia, the rapid thermonuclease test seems to be as insensitive as slide agglutination, ${ }^{5}$ whereas a DNA probe method is currently not cost-effective. The aurease test is rarely used. ${ }^{3}$ The DTC test remains a simple, cheap, rapid, and reliable method available to all diagnostic laboratories. Medical microbiology departments may be deterred from its use (only 22 of 287 UK laboratories performed a DTC test in $1992^{3}$ ) because of the commonly used, but labour intensive, double centrifuga- tion step. ${ }^{69}$ However two recent reports have indicated no loss of sensitivity when the DTC test is performed directly on uncentrifuged blood culture broths. ${ }^{510}$ This finding should encourage laboratories to use the DTC test routinely for the rapid identification of $S$ aureus from positive blood cultures.

We thank Miss $T$ Barnett for her secretarial assistance in preparing this manuscript.

1 Washington JA and the International Collaborative Blood Culture Study Group. An international multicentre study of blood culture practices. Eur $\mathcal{F}$ Clin Microbiol Infect Dis 1992;11:1115-28.

2 Agger WA, Maki DG. Efficacy of direct Gram stain in differentiating staphylococci from streptococci in blood cultures positive for Gram-positive cocci. $\mathcal{f}$ Clin Microbiol 1978;7:111-13.

3 Brown DFJ, Perry SF. Methods used in the United Kingdom for the culture of micro-organisms from blood. $\mathcal{f}$ Clin Pathol 1992;45:468-74.

4 Rossney AS, English LF, Keane CT. Coagulase testing compared with commercial kits for routinely identifying compared with commercial kits for routinely identify

5 Davis TE, Fuller DD, Aeschleman EC. Rapid, direct identification of Staphylococcus aureus and Streptococcus pneumoniae from blood cultures using commercial immunologic kits and modified conventional tests. Diagn Microbiol Infect Dis 1992;15:295-300

6 Hamoudi AC, Hribar MM. Evaluation of a direct identification method for Staphylococcus aureus from blood culture broth. F Clin Microbiol 1988;26:1404-5.

7 Doern GV, Robbie LI. Direct identification of Staphylococcus aureus in blood culture fluid with a commercial latex cus aureus in blood culture fluid with a commercial

8 Rappaport T, Sawyer KP, Nachamkin I. Evaluation of several commercial biochemical and immunologic methods for rapid identification of Gram-positive cocci directly from blood cultures. F Clin Microbiol 1988;26:1335-8.

9 Claxton PM, Mesterton RG. Rapid organism identification from Bactec NR blood culture media in a diagnostic microbiology laboratory. $\mathcal{f}$ Clin Pathol 1994;47:796-8.

10 McDonald CL, Chapmin K. Rapid identification of Staphylococcus aureus from blood culture bottles by a classic 2-hour tube coagulase test. $\mathcal{f}$ Clin Microbiol 1995;33:50-2.

\title{
Bone marrow granulomas in infiltrating lobular breast cancer
}

\author{
P Kettle, D C Allen
}

Laboratory, Belvoir Park Hospital, Belfast P Kettle

\section{Histopathology} Department, Belfast City Hospital, Belfast D C Allen

Correspondence to: Dr P Kettle, Laboratory, Belvoir Park Hospital, Hospital Road, Belfast BT8 8JR

\begin{abstract}
A 50 year old woman with a history of infiltrating lobular breast carcinoma presented with back pain. Bone scan and magnetic resonance imaging were not conclusive. A bone marrow aspirate appeared normal. A routine trephine biopsy specimen showed granulomas but no obvious infiltration by carcinoma. Immunohistochemical staining with epithelial markers demonstrated carcinoma cells in the trephine specimen. This case illustrates the difficulty of detecting infiltrating lobular carcinoma in bone marrow and the value of immunological techniques in this context. It also describes the
\end{abstract}

development of bone marrow granulomas as a response to infiltration by carcinoma. (F Clin Pathol 1997;50:166-168)

Keywords: bone marrow granulomas; lobular breast cancer; immunohistology.

Granuloma formation is well recognised in lymph nodes draining an area of carcinoma. Granuloma formation in bone marrow has a wide differential diagnosis, including granulomatous infections, sarcoid, drug hypersensitivity, lymphoid, and haemopoietic malignancy. ${ }^{1}$ Metastatic carcinoma does not seem to be a widely recognised cause. 


\section{Case report}

A 45 year old woman, with a long psychiatric history, presented in 1991 with a mass in the right breast. Histological examination showed infiltrating lobular carcinoma. A simple mastectomy was performed. She declined further intervention at that stage. In 1993, biopsy of a mass in the left breast showed lobular carcinoma which also involved four axillary nodes. She then had a mastectomy with local radiotherapy followed by five courses of chemotherapy (5-fluorouracil, epirubicin and cyclophosphamide).

In 1995, the patient developed back pain. A bone scan was inconclusive. Magnetic resonance imaging of the pelvis was reported as abnormal but not typical of metastatic disease. Bone marrow was sampled from the posterior superior iliac crest-the findings are given in detail later. The patient was subsequently started on tamoxifen but has not attended regularly for follow up. There was no evidence of tuberculosis or sarcoidosis, and the patient was not on any regular medication.

\section{Pathology}

The bone marrow aspirate was stained routinely with Giemsa. The aspirate was of normal cellularity with no abnormality detected. Immunostaining with epithelial markers by the APAAP technique was negative. The bone marrow trephine specimen was fixed in Bouin's solution, decalcified for 24 hours by adding concentrated formic acid to a dilution of $10 \%$, embedded in paraffin wax, and processed using standard techniques. The haematoxylin and eosin stained section revealed a normocellular marrow. The most striking feature was the presence of non-caseating granulomas (fig 1). These granulomas were composed of epithelioid cells and Langhans giant cells. No tumour cells were evident. A Ziehl-Neelsen stain did not show any acid fast bacilli. However, streptavidin-biotin immunoperoxidase staining showed cells which were positive for cytokeratins $\mathrm{AE}_{1} / \mathrm{AE}_{3}$ and Cam 5.2 (acidic keratins $40-56.5 \mathrm{kD}$, basic keratins $52-67 \mathrm{kD}$ ), and EMA. These positive cells occurred singly throughout the section, in keeping with micrometastases (fig 2). The granulomas were adjacent to the carcinoma cells.

\section{Discussion}

Infiltrating lobular carcinoma of breast is difficult to detect in standard haematoxylin and eosin stained sections of bone marrow. Bitter et $a l^{2}$ distributed 50 core biopsy specimens to three surgical pathologists. Infiltrating lobular carcinoma of breast was correctly identified in only $39 \%$ of positive specimens compared with $88 \%$ for ductal carcinoma. The low detection rate was explained by the difficulty in distinguishing infiltrating lobular carcinoma cells from haemopoietic cells of similar size, and by their bland cytological features and infiltration as single cells with little tissue reaction. The findings in this case would concur and support the suggestion that trephine specimens from patients with infiltrating lobular carcinoma be immunostained routinely with cytokeratin.

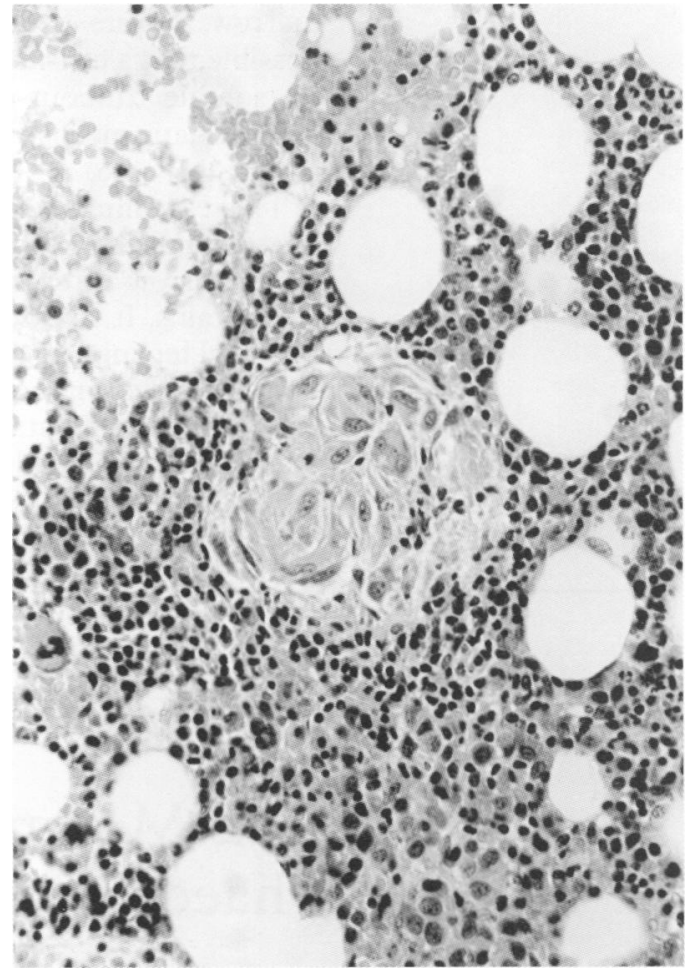

Figure 1 Bone marrow trephine biopsy specimen showing non-caseating granulomas.

Granuloma formation in regional lymph nodes draining carcinoma, especially breast and cervix, is well recognised. ${ }^{3}$ The simultaneous occurrence of granulomas and metastatic carcinoma within the bone marrow is unusual and, to our knowledge, has not been documented previously. No other cause of granuloma formation could be detected in this patient. Quite often, no cause can be established for granuloma formation in the bone

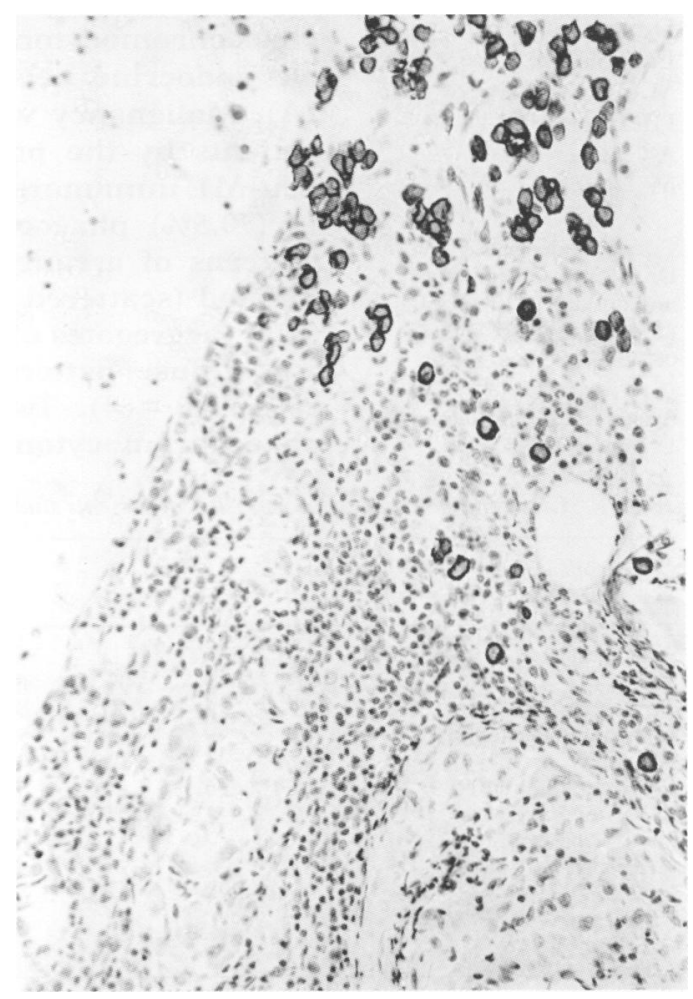

Figure 2 Immunostaining with cytokeratin showing granulomas in relation to positive carcinoma cells. 
marrow despite extensive investigation. It is possible that a cell-mediated immune response to soluble tumour-related antigens is the pathogenetic mechanism for granuloma formation in this case.

This case illustrates the difficulty of demonstrating infiltrating lobular breast carcinoma in bone marrow using routine haematoxylin and eosin stains. It shows the value of immunohistological techniques. Demonstration of infiltration can have implications for prognosis and patient management. ${ }^{4}$ It also describes the formation of epithelioid granulomas in the bone marrow in response to micrometastases of lobular breast carcinoma.

We thank Dr McAleer for permission to report this case.

1 Bain BJ, Clark DM, Lampert IA. In: Bone marrow pathology. London: Blackwell Scientific Publications, 1992:47.

2 Bitter MA, Fiorito D, Corkill ME, Huffer WE, Stemmer SM, Shpall EJ, et al. Bone marrow involvement by lobular carcinoma of the breast cannot be identified reliably by routine histological examination alone. Hum Pathol 1994; 25:781-8.

3 Gregorie HB, Othersen HB, Moore MP. The significance of sarcoid-like lesions in association with malignant neoplasms. Am $\mathcal{F}$ Surg 1962;104:577-86.

4 Untich M, Harbeck N, Eimann W. Micrometastases in bone marrow in patients with breast cancer. $B M F$ 1988;296:290.

\title{
Leu-M1 immunoreactivity and phaeochromocytoma
}

\author{
L Masmiquel, M Castro-Forns, I de Torres, A García, M T Vidal, R Simó
}

\section{Department of Endocrinology, Hospital Universitari Vall d'Hebron, Barcelona, Spain L Masmiquel \\ R Simó}

Department of Pathology

M Castro-Forns

I de Torres

A García

M T Vidal

Correspondence to: Dr R Simó, C/Can Picanyol no 10, Edifici Mimosa, Bajos 2, Sant Cugat del Valles, 08190 Barcelona, Spain.

Accepted for publication 18 January 1995

\begin{abstract}
The aim was to evaluate Leu-M1 immunoreactivity as a prognostic factor in phaeochromocytoma. Anti-Leu-M1 monoclonal antibodies were used to determine the Leu-M1 immunoreactivity in 17 histologically confirmed phaeochromocytomas from 15 patients, using an avidin-biotin technique. Ten patients had a sporadic phaeochromocytoma, and five had multiple endocrine neoplasia type $2 A$ (MEN 2A). Malignancy was diagnosed in three patients by the presence of metastases. Leu-M1 immunoreactivity was shown in $12(70.5 \%)$ phaeochromocytomas. Three patterns of arrangement were observed: isolated (scattered positive cells) $(n=3)$; focal (aggregates of positive cells) $(n=5)$, and diffuse patterns (dispersed positive cells) $(n=4)$. Two cases of malignant phaeochromocytoma were positive (one
\end{abstract}

focal and one isolated pattern). All cases of MEN 2A showed immunoreactivity, although no characteristic pattern was prevalent. A diffuse pattern was observed in all phaeochromocytomas longer than $7 \mathrm{~cm}$. In conclusion, Leu-M1 expression is frequent in phaeochromocytoma. However, Leu-M1 immunoreactivity seems to be useless in predicting malignant behaviour and to be influenced mainly by tumour size.

(f Clin Pathol 1997;50:168-170)

Keywords: Leu-Ml antigen; phaeochromocytoma; multiple endocrine neoplasia.

Phaeochromocytoma is an infrequent tumour derived from chromaffin tissue. It occurs sporadically but in $10 \%$ of cases it is associated

Table 1 Clinicopathological characteristics of patients studied

\begin{tabular}{|c|c|c|c|c|c|c|c|c|c|c|c|c|c|c|c|}
\hline \multirow{2}{*}{$\begin{array}{l}\text { Clinicopathological } \\
\text { characteristic }\end{array}$} & \multicolumn{2}{|c|}{ Case number } & \multirow[b]{2}{*}{3} & \multirow[b]{2}{*}{4} & \multirow[b]{2}{*}{5} & \multirow[b]{2}{*}{6} & \multirow[b]{2}{*}{7} & \multirow[b]{2}{*}{8} & \multirow[b]{2}{*}{$9^{a}$} & \multirow[b]{2}{*}{10} & \multirow[b]{2}{*}{$11^{a}$} & \multirow[b]{2}{*}{$12 B$} & \multirow[b]{2}{*}{$13 B$} & \multirow[b]{2}{*}{14} & \multirow[b]{2}{*}{$15^{a}$} \\
\hline & 1 & 2 & & & & & & & & & & & & & \\
\hline Age (years) & 38 & 33 & 67 & 41 & 48 & 34 & 47 & 33 & 39 & 54 & 28 & 25 & 19 & 58 & 23 \\
\hline Sex & $\mathbf{M}$ & $\mathrm{F}$ & $\mathrm{F}$ & F & F & $\mathbf{M}$ & $\mathbf{M}$ & $\mathbf{M}$ & $\mathrm{F}$ & F & $\mathbf{F}$ & $\mathbf{M}$ & $\mathbf{F}$ & $\mathrm{F}$ & F \\
\hline Type & $\mathbf{S}$ & $\mathbf{S}$ & $\mathrm{S}$ & $\mathbf{S}$ & $\mathbf{S}$ & $\mathbf{S}$ & $\mathbf{S}$ & $\mathbf{S}$ & $\mathbf{S}$ & $\mathbf{S}$ & MEN & MEN & MEN & MEN & MEN \\
\hline Clinical manifestations & + & + & + & + & + & + & + & + & + & + & + & - & + & - & + \\
\hline Size $(\mathrm{cm})$ & 3.5 & 2.7 & 4.5 & 7.8 & 5 & 6.3 & 8 & 6 & 5.5 & 3.5 & 5.4 & $2.5 / 4$ & $9.5 / 3$ & 10 & 4 \\
\hline $\mathrm{CM}(\mu \mathrm{mol} / 24$ hours $)$ & 3.4 & 4.9 & 5.9 & 1.4 & 8.4 & 4.1 & 5.4 & 12 & 1.9 & 22.3 & 16.1 & 19.6 & 1.7 & 6 & 3.8 \\
\hline VMA ( $\mu \mathrm{mol} / 24$ hours) & 62.6 & 58 & 79 & 272 & 110 & 55.7 & 129 & 34 & 56 & 85 & 340 & 359 & 22 & 193 & 40.3 \\
\hline $\begin{array}{l}\text { Leu-M1 pattern } \\
\text { (intensity) }\end{array}$ & $F(++)$ & - & - & $\mathrm{D}(+++$ & - & $F(++)$ & $\mathrm{D}(++)$ & $I(+)$ & - & - & $I(+)$ & $\begin{array}{l}\mathrm{F} / \mathrm{F}(+) \\
++)\end{array}$ & $\begin{array}{l}\mathrm{D} / \mathrm{I}(+++/ \\
++)\end{array}$ & $D(+++)$ & $F(++)$ \\
\hline $\begin{array}{l}\text { Inflammatory } \\
\text { component (Leu-M1 } \\
\text { intensity) }\end{array}$ & - & - & - & - & - & - & + & - & ++ & - & +++ & - & - & - & +++ \\
\hline
\end{tabular}

